\title{
Air pollution and development of asthma, allergy and infections in a birth cohort
}

\author{
M. Brauer*, G. Hoek\#, H.A. Smit ${ }^{\mp}$, J.C. de Jongste ${ }^{+}$, J. Gerritsen ${ }^{\S}$, D.S. Postma ${ }^{f}$, \\ M. Kerkhof** and B. Brunekreef ${ }^{\#}$
}

ABSTRACT: Few studies have addressed associations between traffic-related air pollution and respiratory disease in young children. The present authors assessed the development of asthmatic/allergic symptoms and respiratory infections during the first 4 yrs of life in a birth cohort study $(n=\sim 4,000)$.

Outdoor concentrations of traffic-related air pollutants (nitrogen dioxide $\mathbf{P M}_{2.5}$, particles with a $50 \%$ cut-off aerodynamic diameter of $2.5 \mu \mathrm{m}$ and soot) were assigned to birthplace home addresses with a land-use regression model. They were linked by logistic regression to questionnaire data on doctor-diagnosed asthma, bronchitis, influenza and eczema and to selfreported wheeze, dry night-time cough, ear/nose/throat infections and skin rash. Total and specific immunoglobulin $(\mathrm{Ig}) E$ to common allergens were measured in a subgroup $(n=713)$.

Adjusted odds ratios (95\% confidence intervals) per interquartile pollution range were elevated for wheeze (1.2 (1.0-1.4) for soot), doctor-diagnosed asthma (1.3 (1.0-1.7)), ear/nose/throat infections (1.2 (1.0-1.3)) and flu/serious colds (1.2 (1.0-1.4)). No consistent associations were observed for other end-points. Positive associations between air pollution and specific sensitisation to common food allergens (1.6 (1.2-2.2) for soot), but not total IgE, were found in the subgroup with IgE measurements.

Traffic-related pollution was associated with respiratory infections and some measures of asthma and allergy during the first 4 yrs of life.

KEYWORDS: Air pollution, allergy, asthma, respiratory infections, vehicle emissions

M any studies have demonstrated that exposure to outdoor air pollutants can exacerbate pre-existing asthma [1, 2], but it is unclear whether outdoor air pollutants increase the incidence of asthma or allergic diseases in children. While a limited number of studies have described associations between asthma and ozone [3, 4], there is growing evidence that air pollutants specifically associated with traffic exposure may be of greater importance to asthma and allergic disease development [5-18].

In particular, a number of recent studies have reported associations between concentrations of air pollution arising directly from motor vehicle emissions and asthma or asthmatic symptoms [5, 6]. Cross-sectional studies have found lung function to be reduced [7] or the prevalence of respiratory symptoms [8-14] to be elevated for individuals living or attending schools close to high-traffic roads. Several case-control studies have also found associations between measures of traffic intensity and respiratory outcomes, such as incidence of wheeze [14] and asthma [15] or hospitalisation for wheezing bronchitis (4-48month-old children) [16] or for asthma (children $<5$ yrs of age) [17]. Despite the fact that the majority of these studies relied on distance to major roads or on measures of traffic intensity as a proxy for exposure to air pollutants, it is suggested that living near busy roads leads to adverse respiratory health effects. A more limited number of studies have included direct measurements or modelled concentrations of air pollution. For example, two recent analyses [18, 19] suggest associations between asthma and several measures of highway pollutants in California, USA, and between asthma and prevalence of cough and bronchitis (but not with atopy in

For editorial comments see page 825 .

This article has supplementary material accessible from www.erj.ersjournals.com

STATEMENT OF INTEREST: None declared.

AFFILIATIONS

*University of British Columbia,

School of Occupational and

Environmental Hygiene, Vancouver, BC, Canada

\#Utrecht University, Institute for Risk Assessment Sciences and Julius Centre for Health Sciences and Primary Care, University Medical Centre Utrecht, Utrecht,

'Centre for Prevention and Health Services Research, National Institute for Public Health and the Environment (RIVM), Bilthoven, ${ }^{+}$Dept of Paediatrics, Division of Respiratory Medicine, Erasmus Medical Centre - Sophia Children's Hospital, Erasmus University, Rotterdam, and

${ }^{\S}$ Depts of Paediatric Respiratory Medicine

${ }^{f}$ Pulmonology, and **Epidemiology, University of Groningen, Groningen, The Netherlands

CORRESPONDENCE M. Brauer: University of British Columbia, School of Occupational and Environmental Hygiene 2206 East Mall, Vancouver BC V6T1Z3, Canada Fax: 16048229588

E-mail: brauer@interchange.ubc.ca

Received: June 262006 Accepted after revision: December 252006

\section{SUPPORT STATEMENT}

The authors have no competing

interests to declare. The present study was funded by NOVEM grant 310100 / 0002, grants from the European Union (ENV4-CT97-0506), the Netherlands Asthma Fund (94.27; Leusden) the Ministry of the Environment (The Hague), ZorgOnderzoek Nederland

(The Hague) and the National Institute of Public Health and the Environment (Bilthoven all The Netherlands). The exposure assessment (Traffic Related Air Pollution and Childhood Asthma) component was supported by the EU grant ENV4-CT0506.

European Respiratory Journal Print ISSN 0903-1936

Online ISSN 1399-3003 
children) in a cross-sectional study in Germany [11], in which air pollution concentrations were estimated for all home and school addresses. A study that used an emissions model to estimate exposures [20] showed an association between several traffic-related pollutants and current wheeze or asthma, but not with allergic sensitisation. A series of studies completed in the Netherlands indicated that children living near roads with high levels of truck traffic exhibited lower lung function and more chronic respiratory symptoms compared with children living on roads with less truck traffic [21]. A similar relationship was found between measures of respiratory health of schoolchildren with levels of traffic-related air pollutants, especially those specific to trucks, measured in their schools $[22,23]$. Several studies [24-26] have also suggested that trafficrelated air pollution may be associated with increased respiratory symptoms in young children, although data are lacking regarding associations with respiratory infections, especially from cohort studies.

The present authors previously reported the relationship between traffic-related air pollution and the development of asthmatic symptoms, allergic diseases and respiratory infections at age 2 yrs in a birth cohort study in the Netherlands [26]. In that study [26], exposure to air pollution was estimated for an existing birth cohort study, i.e. Prevention and Incidence of Asthma and Mite Allergy (PIAMA) [27, 28]. A unique aspect of the study was the use of individual exposure estimates for each cohort member derived from a land-use regression model [29-31]. Individual estimates of exposure to traffic-related air pollution were computed using a validated model based on geographic data and air pollution measurements of nitrogen dioxide $\left(\mathrm{NO}_{2}\right)$, particles with a $50 \%$ cut-off aerodynamic diameter of $2.5 \mu \mathrm{m}$ (PM2.5) and filter reflectance, a measure of particulate elemental carbon (soot). Significant positive associations at $2 \mathrm{yrs}$ of age between traffic-related air pollutants and wheezing were reported. A related study conducted in Munich, Germany [32], using the same exposure assessment approach found associations between traffic-related air pollutants and cough, but concluded that the young age of the cohort ( 2 yrs) precluded conclusions related to asthma.

Although the present authors' previous analysis [26] suggested associations of traffic-related air pollution with asthma and respiratory infections at 2 yrs of age, the implication of wheeze or asthma reported at such a young age is somewhat unclear. Therefore, the previous analysis of the same birth cohort was extended in order to assess the association between individual level estimates of exposure to traffic-related air pollution with respiratory symptoms and measures of allergy reported at 4 yrs of age. At 4 yrs reporting of asthma and symptoms of wheeze have a clearer implication, although diagnoses of asthma are still tentative. In addition, the association between air pollution and objectively measured sensitisation to common allergens was analysed in a subset of the cohort.

\section{METHODS}

\section{Study population}

The PIAMA prospective birth cohort study had an initial enrolment of 4,146 children recruited during the second trimester of pregnancy from a series of communities of variable size in the Netherlands [27, 28]. The study protocol was approved by the institutional review board of each participating institute and informed written consent was obtained from all participants. At inception, the study included a group of 855 high-risk children who were randomly allocated to control and intervention groups in which allergen-free mattress covers were used. In addition, the study population included a natural history group of 3,291 children, including 472 high-risk children. High-risk children were identified before birth by obtaining information regarding self-reported asthma or reporting of physician-diagnosed allergy to house dust, house dust mites, pets or pollen in the expecting mothers. The current analysis included all children (natural history and intervention) who remained in the cohort at 4 yrs of age.

\section{Exposure assessment}

Details of exposure assessment have been reported elsewhere [29-31, 33]. Briefly, air pollution concentrations at the home address at birth were calculated by a model combining air pollution measurements with a Geographic Information System (GIS). Fine particles (defined according to PM2.5), $\mathrm{NO}_{2}$ and soot (determined as the reflectance of the PM2.5 filters) were measured at 40 sites designed to capture the maximum variability in pollution from traffic sources. At each location, measurements were conducted for four 2-week periods dispersed throughout $1 \mathrm{yr}$ (March 1, 1999 to April 20, 2000; corresponding to a period when the cohort was on average $2-3$ yrs old) and then adjusted for temporal trends to calculate long-term average concentrations. The adjustment consisted of: 1) calculation of an annual average for a single reference site where 14-day integrated samples were made for the entire study period; 2) calculation for the reference site of the difference between the annual average and the measurements for each 2-week sampling period at all other sites; 3) addition of the difference for each sampling period to the measured concentrations at each site during this period; and 4) calculation of an annual average from the adjusted values [31]. Therefore, this approach to exposure assessment emphasises spatial contrasts in long-term average concentrations during the first 4 yrs of life, since it was hypothesised that it would have greater relevance for the development of chronic respiratory disease than short-term temporal variations in concentrations. At the same locations, GIS data were also collected regarding traffic in the vicinity of each monitoring location. Regression models were developed to relate the annual average concentrations measured at the 40 monitoring sites with the GIS variables; e.g. the number of high-traffic roads within a $250-\mathrm{m}$ radius of a location, the presence of a major road within a distance of $50 \mathrm{~m}$, the density of buildings within a 300-m radius and an indicator for the region of the country were used in the model to predict soot concentrations. Models with similar variables describing traffic intensity were developed for PM2.5 and $\mathrm{NO}_{2}$. These models explained 73, 81 and $85 \%$ of the variability in the annual average concentrations for PM2.5, soot and $\mathrm{NO}_{2}$, respectively [29-31]. These models were applied to the same GIS variables measured at the home addresses of each cohort member in order to obtain individual long-term ambient air pollution exposure estimates. Exposure was assessed for the address at birth to reflect the present authors' interest in the effects of early life exposures on respiratory health. 


\section{Questionnaire data}

From questionnaires completed by the parents of cohort members, the same health outcomes as in the present authors' previous analysis [26] were selected to describe symptom frequencies and physician diagnoses for indicators of asthma, allergic disease and infections (table 1). Questionnaires were completed during pregnancy, at the time the child was 3 months old and at the time of the child's first, second, third and fourth birthday. Each questionnaire contained a core set of questions that were supplemented with additional questions as the cohort aged, i.e. additional variables describing wheeze and asthma development were added to the current analysis (table 2). The online supplementary material lists symptoms, diagnoses, exact questions, definitions of early-onset transient, late-onset and persistent wheeze, and a definition of "possible/probable asthma" based upon reported symptoms and other conditions. The definition was developed in the PIAMA project according to guidelines of the Dutch General Practitioners Association, with the relevant questions identical to those of the International Study of Asthma and Allergies in Childhood [34]. Analyses were conducted with the same potential confounding variables as in the present authors' previous study (table 3). The data for these variables were

\begin{tabular}{|c|c|c|c|}
\hline TABLE 1 & \multicolumn{3}{|c|}{$\begin{array}{l}\text { Prevalence of selected health outcomes at } 4 \text { yrs } \\
\text { of age in the full cohort and of sensitisation to } \\
\text { specific allergens based on immunoglobulin } \\
\text { (lg)E measurement in a cohort subset }\end{array}$} \\
\hline \multicolumn{2}{|l|}{ Variable } & Subjects & Prevalence \% \\
\hline \multicolumn{2}{|l|}{ Wheeze } & 429 (3528) & 12.2 \\
\hline \multicolumn{2}{|c|}{ Doctor-diagnosed asthma } & 144 (3533) & 4.1 \\
\hline \multicolumn{2}{|c|}{ Dry cough at night with/without cold } & $782(3537)$ & 22.1 \\
\hline \multicolumn{2}{|c|}{ Doctor-diagnosed bronchitis } & $214(3501)$ & 6.1 \\
\hline \multicolumn{2}{|l|}{ Itchy rash } & 906 (3537) & 25.6 \\
\hline \multicolumn{2}{|c|}{ Doctor-diagnosed eczema } & $503(3518)$ & 14.3 \\
\hline \multicolumn{2}{|c|}{ Ear/nose/throat infections } & 1047 (3549) & 29.5 \\
\hline \multicolumn{2}{|c|}{ Doctor-diagnosed flu, serious cold } & $760(3521)$ & 21.6 \\
\hline \multicolumn{2}{|c|}{ Any positive sp $\lg E$} & $277(713)$ & 38.8 \\
\hline \multicolumn{2}{|c|}{ Any indoor positive sp IgE } & $118(713)$ & 16.5 \\
\hline \multicolumn{2}{|c|}{ Cat allergen positive } & $44(708)$ & 6.2 \\
\hline \multicolumn{2}{|c|}{ Dog allergen positive } & $26(706)$ & 3.7 \\
\hline \multicolumn{2}{|c|}{ Dust mite allergen positive } & $101(713)$ & 14.2 \\
\hline \multicolumn{2}{|c|}{ Any outdoor positive $s p \lg E$} & $57(698)$ & 8.2 \\
\hline \multicolumn{2}{|c|}{ Dactylium positive } & $47(698)$ & 6.7 \\
\hline \multicolumn{2}{|c|}{ Birch positive } & $16(696)$ & 2.3 \\
\hline \multicolumn{2}{|c|}{ Alternaria positive } & $11(680)$ & 1.7 \\
\hline \multicolumn{2}{|c|}{ Any food positive sp IgE } & $194(689)$ & 28.1 \\
\hline \multicolumn{2}{|c|}{ Milk allergen positive } & $182(689)$ & 26.4 \\
\hline \multicolumn{2}{|c|}{ Egg allergen positive } & $51(681)$ & 7.5 \\
\hline \multicolumn{2}{|c|}{ Total IgE $>100 \mathrm{IU} \cdot \mathrm{mL}^{-1}$} & $186(713)$ & 26.1 \\
\hline
\end{tabular}

Data for subjects are presented as number presenting with symptom, condition or positive IgE reaction to a specific allergen; with the total number of subjects, evaluated based on completion of a questionnaire at 4 yrs and allergy testing where applicable, given in parentheses. sp: Specific serum. All outcomes refer to incidence or diagnoses within the previous 12 months, the period between 3-4 yrs of age (see online supplementary material). selected, from the earliest questionnaire available, to coincide with the estimated exposures for birth addresses. Details are presented in the legend of table 3.

\section{Sensitisation data}

A blood sample was taken at 4 yrs of age in a total of 713 children who also had a home visit at 3 months of age and who completed the year 4 questionnaire. Consent for blood sampling was obtained from all parents. Blood sampling was offered to children participating in the intervention study and those children in the natural history part of the cohort who had had a home visit at 3 months of age [35]. Total and specific immunoglobulin (Ig)E levels for house dust mite, cat, dog, Dactylium, birch pollen, Alternaria, egg and milk were measured. IgE was determined by a radioallergosorbent testlike method according to the standard operating procedure used at the Sanquin Laboratories (Amsterdam, The Netherlands). Positive reactions were defined as those with IgE levels $>0.35 \mathrm{IU} \cdot \mathrm{mL}^{-1}$. Sensitisation was defined as elevated IgE to any of the measured allergens, any indoor allergens, any of the outdoor allergens or any of the food allergens.

\section{Statistical analysis}

The association between exposure and health outcomes was analysed by multiple logistic regression, with adjustment for confounding variables. Sensitivity analyses were conducted for the subset of the cohort who remained at the birth address at 4 yrs of age and those who remained at the same address since their first birthday. While the primary analysis focused on the relationship between health outcomes at 4 yrs of age and risk factors in the first year of life, a separate analysis was also conducted, including risk factors information (gas stove, pets, siblings and home dampness) from the questionnaire at 4 yrs. Odds ratios (OR) are presented for an interquartile range increase in air pollutant concentrations equivalent to $3.3 \mu \mathrm{g} \cdot \mathrm{m}^{-3}$ for PM2.5, $0.58 \times 10^{-5} \mathrm{~m}^{-1}$ for soot (corresponding to $0.84 \mu \mathrm{g} \cdot \mathrm{m}^{-3}$ elemental carbon) [30] and $10.6 \mu \mathrm{g} \cdot \mathrm{m}^{-3}$ for $\mathrm{NO}_{2}$. A stratified analysis was also performed on the subset of the population with sensitisation measurements in order to assess whether the association between air pollution and symptoms was stronger in children who were sensitised.

\section{RESULTS}

\section{Prevalence of health outcomes}

At the time of the first questionnaire (3 months of age), 212 children had dropped out of the study $(n=3,934)$. At 1 yr the cohort size was 3,745, and was 3,730 at 2 yrs. At 4 yrs, data were collected from 3,538 children, corresponding to $85.3 \%$ retention. Of those children remaining in the study population at 4 yrs of age, $608(17.2 \%)$ were in the intervention arm, representing a slightly lower proportion $(19.3 \%)$ than at the initiation of the cohort. The main reasons for dropout were loss of interest, lack of available time and subjects moving and becoming untraceable. Although complete data was not available for any of the covariates, subjects who dropped out of the study (see online supplementary material) were more likely to have allergic mothers, parents with less than a medium level of professional education, mothers who smoked during pregnancy and were more likely to live in homes with a smoker, mold and an unvented gas heater. These subjects also were less likely to have been breastfed at 3 months of age, were 


\begin{tabular}{|c|c|c|c|}
\hline TABLE 2 & \multicolumn{3}{|c|}{$\begin{array}{l}\text { Cumulative incidence of selected health } \\
\text { outcomes in the full cohort at } 4 \text { yrs of age. }\end{array}$} \\
\hline \multicolumn{2}{|l|}{ Variable } & Subjects & $\begin{array}{l}\text { Cumulative } \\
\text { incidence \% }\end{array}$ \\
\hline \multicolumn{2}{|c|}{ Wheeze ever $\#$} & $960(3530)$ & 27.2 \\
\hline \multicolumn{2}{|c|}{ Doctor-diagnosed asthma ever ${ }^{\#}$} & $258(3534)$ & 7.3 \\
\hline \multicolumn{2}{|c|}{ Possible asthma } & $52(3549)$ & 1.5 \\
\hline \multicolumn{2}{|c|}{ Probable asthma } & $516(3549)$ & 14.5 \\
\hline \multicolumn{2}{|c|}{ Possible or probable asthma } & 568 (3549) & 16.0 \\
\hline \multicolumn{2}{|c|}{ Early wheeze } & $843(3872)$ & 21.8 \\
\hline \multicolumn{2}{|c|}{ Late wheeze } & 72 (3872) & 1.9 \\
\hline \multicolumn{2}{|c|}{ Persistent wheeze } & 333 (3872) & 8.6 \\
\hline \multicolumn{2}{|c|}{ Early frequent wheeze } & $353(3872)$ & 9.1 \\
\hline \multicolumn{2}{|c|}{ Late frequent wheeze } & 47 (3872) & 1.2 \\
\hline \multicolumn{2}{|c|}{ Persistent frequent wheeze } & 58 (3872) & 3.0 \\
\hline \multicolumn{2}{|c|}{ Doctor-diagnosed bronchitis ever" } & $628(3481)$ & 18.0 \\
\hline \multicolumn{2}{|c|}{ Doctor-diagnosed eczema ever ${ }^{\#}$} & $974(3470)$ & 28.1 \\
\hline \multicolumn{2}{|c|}{ Itchy rash/eczema } & 1438 (3873) & 37.1 \\
\hline \multicolumn{2}{|c|}{ Allergic ${ }^{\#}$} & $106(3542)$ & 3.0 \\
\hline
\end{tabular}

Data for subjects are presented as number presenting with symptom or condition (total number of subjects evaluated). The outcome is based upon all questionnaires (see online supplementary material). \#: Outcomes refer to cumulative incidence or diagnoses within the first 4 yrs of life based on the questionnaire at 4 yrs.

born to younger mothers and were more likely to live in the western part of the Netherlands. Finally, those subjects that dropped out had higher estimated exposures to traffic-related air pollutants.

Health outcomes are presented separately for those variables defined by the questionnaire or sensitisation measurements conducted at $4 \mathrm{yrs}$ of age (and referring to diagnoses and symptoms experienced in the fourth year of life; table 1) and cumulative indicators of disease (table 2) based on the experiences during the first $4 \mathrm{yrs}$ of life. The highest prevalence of symptoms at 4 yrs of age was found for ear/ nose/throat infections (table 1). The prevalence of wheeze was about three times higher than doctor-diagnosed asthma.

Of the 3,538 subjects who completed the questionnaire at 4 yrs, $1,836(52 \%)$ subjects resided in the same house since birth and $2,497(71 \%)$ resided in the same house from 1-4 yrs of age. Prevalence of health outcomes were very similar for these subsets of the cohort (data not shown) relative to the full cohort. A slightly elevated prevalence for early wheeze was observed in the subset remaining at the birth address $(23.6 \%)$ and those residing at the same address from age $1 \mathrm{yr}(22.7 \%)$ relative to the full cohort $(21.8 \%)$.

The prevalence of sensitisation to any allergen was high and primarily due to sensitisation to food allergens (28.1\%), with relatively few children $(8.2 \%)$ being sensitised to outdoor allergens. It is noteworthy that this prevalence cannot be compared with the very low prevalence of self-reported allergy in table 2, since the sensitisation data were available for a subgroup of children in which children born to allergic mothers were overrepresented [35]. As a result, the subpopu-

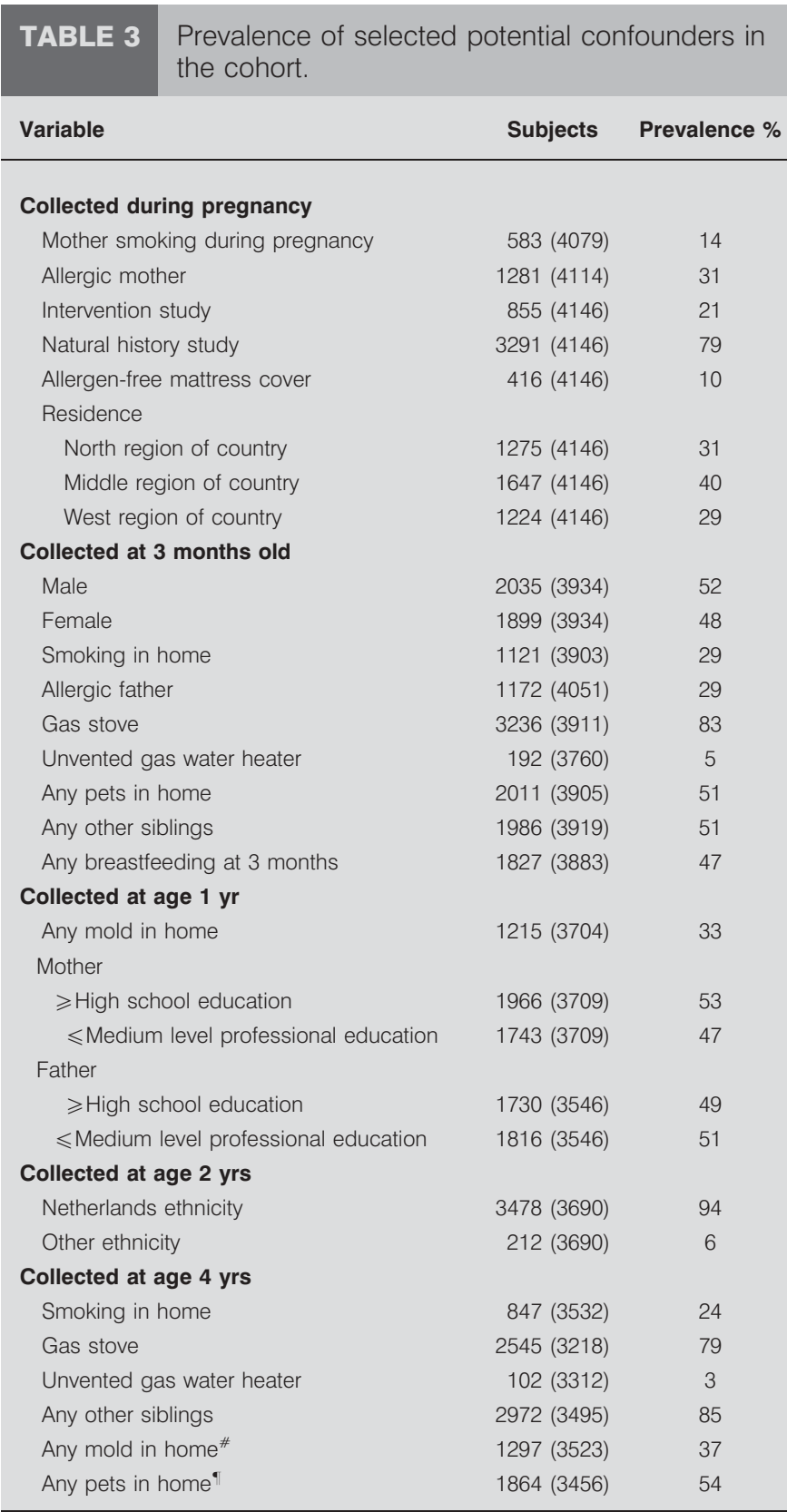

Data for subjects are presented as number with the characteristic (total number of subjects evaluated). ${ }^{*}$ : Includes presence of mould, water damage and visible moisture in any of four specified rooms; ${ }^{\bullet}$ : refers to cats, dogs, rodents or birds. Prevalence of pets, gas water heater and gas stove at 4 yrs may not be completely accurate due to incomplete data (questions are asked only for cases in which there was a change since the previous year's questionnaire), which leads to a slight increase in missing values.

lation with blood sampling differed from the full cohort in some respects. The online supplementary material presents prevalence of the health outcomes and potential confounding variables for this subpopulation. At 4 yrs of age, these children reported significantly higher prevalence of wheeze, physiciandiagnosed asthma, cough, bronchitis, itchy rash and physiciandiagnosed eczema in the previous 12 months than did the full cohort. Further, this subpopulation was more likely to be 
female, to have older siblings, to have been breastfed for $\geqslant 3$ months and to have a lower prevalence of smoking during pregnancy, smoking in the home, pets in the home and fathers with less than a medium level of professional education. These differences suggest that the subset was likely to be at greater risk of developing allergic disease but were more aware of relevant risk factors and took steps to minimise such risks. The tendency for families with children at increased risk for the development of allergic diseases, based on maternal allergy status, to avoid known risk factors has been reported previously for this same cohort [27].

\section{Prevalence of potential confounders}

Table 3 lists the distribution of potential confounders in the study population. Prevalence of smoking was 24\%. Most households used gas for cooking (79\%). Unvented gas water heaters were present in only 3\% of the homes. Virtually all children were of Dutch ethnicity. There were no major differences in the prevalence of potential confounders between the full cohort and the subset who remained at the birth address.

\section{Exposure to air pollutants}

Exposures were successfully calculated for the home addresses of 3,532 of the 3,538 children (99.8\%) and are summarised in table 4 . For six subjects, exposures were not calculated due to an inability to geo-code their home addresses or an error in the GIS calculation of one variable. The exposure estimates for the different pollutants were very highly correlated. The correlation between soot and $\mathrm{NO}_{2}$ was 0.96 , and between soot and $\mathrm{PM} 2.5$, 0.97. The correlation between $\mathrm{NO}_{2}$ and PM2.5 was 0.93 . There were only very minor differences in exposure $(<2 \%$ for median or 90th percentile exposures) between the full cohort and those subsets who remained at the birth address. Given the similarities in exposure, prevalence of potential confounders and health outcomes between the full cohort and those who did not move, no sensitivity analyses of the associations between air pollution exposure and the main outcomes were conducted for these groups. Such sensitivity analyses were

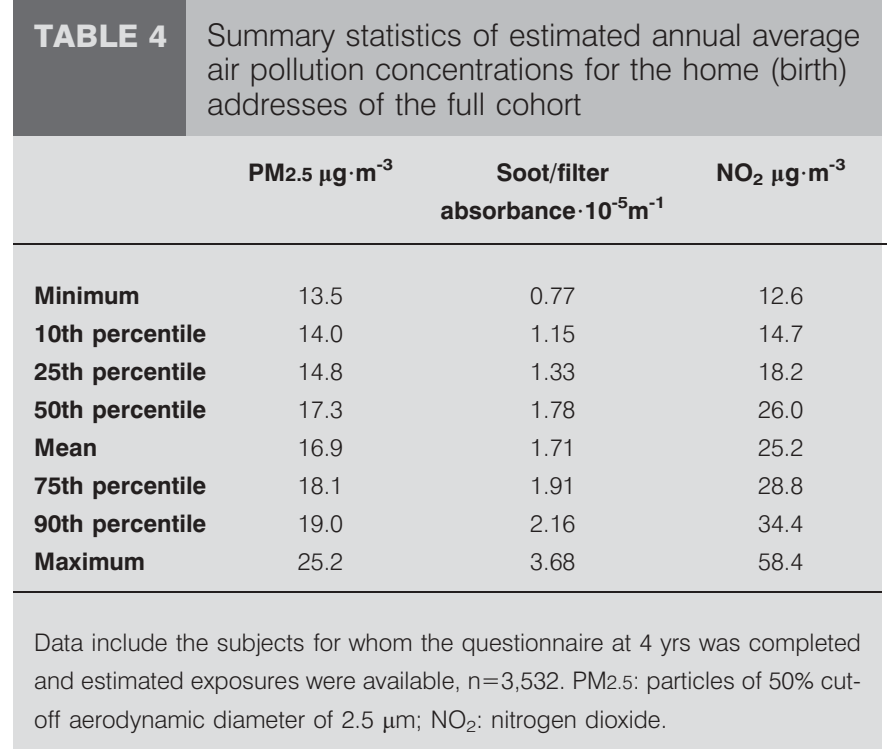

performed for the sensitisation relationships given the small size of the group with sensitisation measures.

\section{Associations between air pollution exposure and health outcomes}

The association between exposure to air pollution and development of respiratory symptoms and sensitisation is reported in tables 5-7. Before adjustment for confounders, air pollution was associated with increased incidence of wheeze, dry cough at night, ear/nose/throat infections and flu/serious colds (table 5), based on responses referring to the fourth year of life only. After adjustment for the full set of confounders, the associations between air pollution and increased risk of wheeze (e.g. OR (95\% confidence interval (CI)) 1.18 (0.981.41) per interquartile range increase in soot), ear/nose/throat infections (e.g. 1.15 (1.01-1.31) for soot) and flu/serious colds (e.g. 1.18 (1.02-1.36) for soot) remained elevated, but the OR for dry cough at night was somewhat decreased (e.g. 1.13 (0.971.30) for soot). Sample sizes for the adjusted OR were reduced due to incomplete questionnaires for some of the confounding variables. When analyses were restricted to adjustment for early-life risk factors, results were similar with somewhat elevated OR for ear/nose/throat infections (e.g. 1.16 (1.03-1.31) for soot), flu/serious colds (e.g. 1.19 (1.04-1.37) for soot) and physician-diagnosed asthma (e.g. 1.30 (0.98-1.71) for soot; table 5).

Sensitisation to any common allergen was positively associated with air pollution, both before and after adjustment, with substantially elevated ORs (e.g. adjusted OR (95\% CI) 1.45 (1.11-1.91) for soot; table 6). The association between sensitisation and air pollution was only present for food allergens. As mentioned previously, this subset was composed of children with higher familial risk of allergy but who lived in lower-risk home environments. Air pollution exposures for the subset did not differ from those of the full cohort.

Table 7 presents the results of analyses in which information from different years was combined to produce cumulative measures of disease status. In the unadjusted analysis, significant associations were found between air pollution exposure and any reporting of wheeze (wheeze ever) and with probable asthma, while there were also indications of associations with physician-diagnosis of asthma and with persistent wheeze. In the adjusted analysis, the sample size was substantially reduced, because only those subjects who completed all questionnaires during the 4 yrs of follow-up were included $(n=\sim 2,500)$. After adjusting for potential confounders, the relationship with wheeze ever remained significant (e.g. OR (95\% CI) 1.18 (1.04-1.34) for soot) and a significant association with physician-diagnosis of asthma was observed (e.g. OR (95\% CI) 1.26 (1.02-1.56) for soot). The association with probable asthma observed in the unadjusted analysis disappeared, while a relationship with early-onset transient wheeze emerged. Associations with persistent wheeze remained borderline significant. No associations were found with doctor diagnosis of bronchitis, eczema or reported itchy rash.

Symptom ORs for nonsensitised and sensitised children were for the birth addresses, sensitivity analyses were also conducted very similar. Since air pollution exposures were assessed only 
TABLE 5 Association between exposure to air pollution and infections, asthmatic and allergic symptoms at 4 yrs of age for the full cohort based on recent and early-life risk factors

\begin{tabular}{|c|c|c|c|c|c|c|}
\hline & \multicolumn{6}{|c|}{ OR $(95 \% \mathrm{Cl})$} \\
\hline & Unadjusted & Subjects $n$ & Adjusted $^{\#}$ & Subjects $\mathrm{n}$ & Adjusted-early life & Subjects $n$ \\
\hline \multicolumn{7}{|l|}{ Wheeze } \\
\hline PM2.5 & $1.20(1.02-1.42)^{*}$ & 3522 & $1.23(1.00-1.51)^{*}$ & 2577 & $1.20(0.99-1.46)$ & 2825 \\
\hline Soot & $1.19(1.03-1.38)^{*}$ & 3522 & $1.18(0.98-1.41)$ & 2577 & $1.18(1.00-1.40)^{*}$ & 2825 \\
\hline $\mathrm{NO}_{2}$ & $1.14(0.99-1.31)$ & 3522 & $1.13(0.95-1.34)$ & 2577 & $1.16(0.98-1.36)$ & 2825 \\
\hline \multicolumn{7}{|c|}{$\begin{array}{l}\text { Doctor-diagnosed } \\
\text { asthma }\end{array}$} \\
\hline PM2.5 & $1.21(0.92-1.60)$ & 3527 & $1.15(0.82-1.62)$ & 2575 & $1.32(0.96-1.83)$ & 2826 \\
\hline Soot & $1.23(0.97-1.56)$ & 3527 & $1.15(0.85-1.55)$ & 2575 & $1.30(0.98-1.71)$ & 2826 \\
\hline $\mathrm{NO}_{2}$ & $1.20(0.96-1.51)$ & 3527 & $1.12(0.84-1.49)$ & 2575 & $1.29(0.99-1.69)$ & 2826 \\
\hline \multicolumn{7}{|c|}{ Dry cough at night } \\
\hline PM2.5 & $1.19(1.04-1.36)^{*}$ & 3531 & $1.11(0.94-1.31)$ & 2578 & $1.14(0.98-1.33)$ & 2830 \\
\hline Soot & $1.19(1.06-1.34)^{*}$ & 3531 & $1.13(0.97-1.30)$ & 2578 & $1.14(1.00-1.31)^{\star}$ & 2830 \\
\hline $\mathrm{NO}_{2}$ & $1.17(1.05-1.30)^{*}$ & 3531 & $1.11(0.97-1.28)$ & 2578 & $1.11(0.97-1.26)$ & 2830 \\
\hline \multicolumn{7}{|c|}{$\begin{array}{l}\text { Doctor-diagnosed } \\
\text { bronchitis }\end{array}$} \\
\hline PM2.5 & $0.96(0.77-1.21)$ & 3496 & $0.88(0.66-1.18)$ & 2559 & $0.86(0.66-1.11)$ & 2807 \\
\hline Soot & $0.98(0.80-1.20)$ & 3496 & $0.90(0.69-1.16)$ & 2559 & $0.88(0.69-1.11)$ & 2807 \\
\hline $\mathrm{NO}_{2}$ & $0.97(0.80-1.17)$ & 3496 & $0.90(0.70-1.15)$ & 2559 & $0.89(0.71-1.12)$ & 2807 \\
\hline \multicolumn{7}{|c|}{ Ear/nose/throat } \\
\hline PM2.5 & $1.11(0.98-1.25)$ & 3543 & $1.13(0.98-1.31)$ & 2587 & $1.17(1.02-1.34)^{*}$ & 2839 \\
\hline Soot & $1.12(1.00-1.24)^{\star}$ & 3543 & $1.15(1.01-1.31)^{*}$ & 2587 & $1.16(1.03-1.31)^{*}$ & 2839 \\
\hline $\mathrm{NO}_{2}$ & $1.13(1.03-1.25)^{*}$ & 3543 & $1.17(1.03-1.32)^{*}$ & 2587 & $1.18(1.05-1.32)^{*}$ & 2839 \\
\hline \multicolumn{7}{|c|}{ Doctor-diagnosed } \\
\hline \multicolumn{7}{|c|}{ flu/serious cold } \\
\hline PM2.5 & $1.17(1.02-1.33)^{*}$ & 3515 & $1.21(1.02-1.42)^{*}$ & 2572 & $1.25(1.07-1.46)^{\star}$ & 2820 \\
\hline Soot & $1.16(1.03-1.30)^{*}$ & 3515 & $1.18(1.02-1.36)^{*}$ & 2572 & $1.19(1.04-1.37)^{\star}$ & 2820 \\
\hline $\mathrm{NO}_{2}$ & $1.13(1.01-1.27)^{\star}$ & 3515 & $1.14(1.00-1.31)$ & 2572 & $1.17(1.02-1.33)^{*}$ & 2820 \\
\hline \multicolumn{7}{|c|}{ Itchy rash } \\
\hline PM2.5 & $1.07(0.95-1.22)$ & 3531 & $0.96(0.82-1.11)$ & 2582 & $0.98(0.85-1.14)$ & 2832 \\
\hline Soot & $1.06(0.95-1.18)$ & 3531 & $0.94(0.82-1.08)$ & 2582 & $0.97(0.85-1.10)$ & 2832 \\
\hline $\mathrm{NO}_{2}$ & $1.05(0.95-1.17)$ & 3531 & $0.95(0.83-1.08)$ & 2582 & $0.97(0.86-1.10)$ & 2832 \\
\hline \multicolumn{7}{|c|}{$\begin{array}{l}\text { Doctor-diagnosed } \\
\text { eczema }\end{array}$} \\
\hline PM2.5 & $1.10(0.97-1.25)$ & 3464 & $1.00(0.83-1.21)$ & 2571 & $0.98(0.82-1.17)$ & 2819 \\
\hline Soot & $1.09(0.98-1.22)$ & 3464 & $0.99(0.84-1.17)$ & 2571 & $0.97(0.83-1.14)$ & 2819 \\
\hline $\mathrm{NO}_{2}$ & $1.06(0.95-1.17)$ & 3464 & $1.00(0.85-1.17)$ & 2571 & $0.97(0.83-1.14)$ & 2819 \\
\hline
\end{tabular}

OR: odds ratio; $\mathrm{Cl}$ : confidence interval; PM2.5: particles with a 50\% cut-off aerodynamic diameter of $2.5 \mu \mathrm{m}$; $\mathrm{NO}_{2}$ : nitrogen dioxide. ${ }^{*}$ : Adjusted for confounders in table 3 and mothers' age at time of birth, but not region; ': adjusted for confounders in table 3 from earliest year available (instead of at 4 yrs) i.e. smoking in the home, gas stove, unvented gas water heater, any other siblings and any mould or pets in home. ORs are calculated for an interquartile range increase in annual average concentration at birth address. *: Significant association, $\mathrm{p}<0.05$.

by restricting to those subjects who remained at the birth address until 4 yrs of age. Adjusted ORs for sensitisation to food allergens were somewhat reduced, but were still greatly elevated for those who had never moved, and were similar to the full cohort for those who had not moved from 1 yr of age.

\section{DISCUSSION}

In the present prospective cohort study, several positive associations of traffic-related air pollutants with wheeze, asthma diagnosis, respiratory infections and sensitisation to food allergens were observed. These findings extend observations made at 2 yrs of age in the same cohort [26]. ORs were not altered to any great extent by the inclusion of potential confounding variables in the regression models or in sensitivity analyses that evaluated the inclusion of confounders measured early rather than later in life. The present authors' indications of associations between exposure to traffic-related air pollutants and respiratory symptoms in children are consistent with results reported elsewhere, although few cohort studies to date have evaluated effects on young children. 


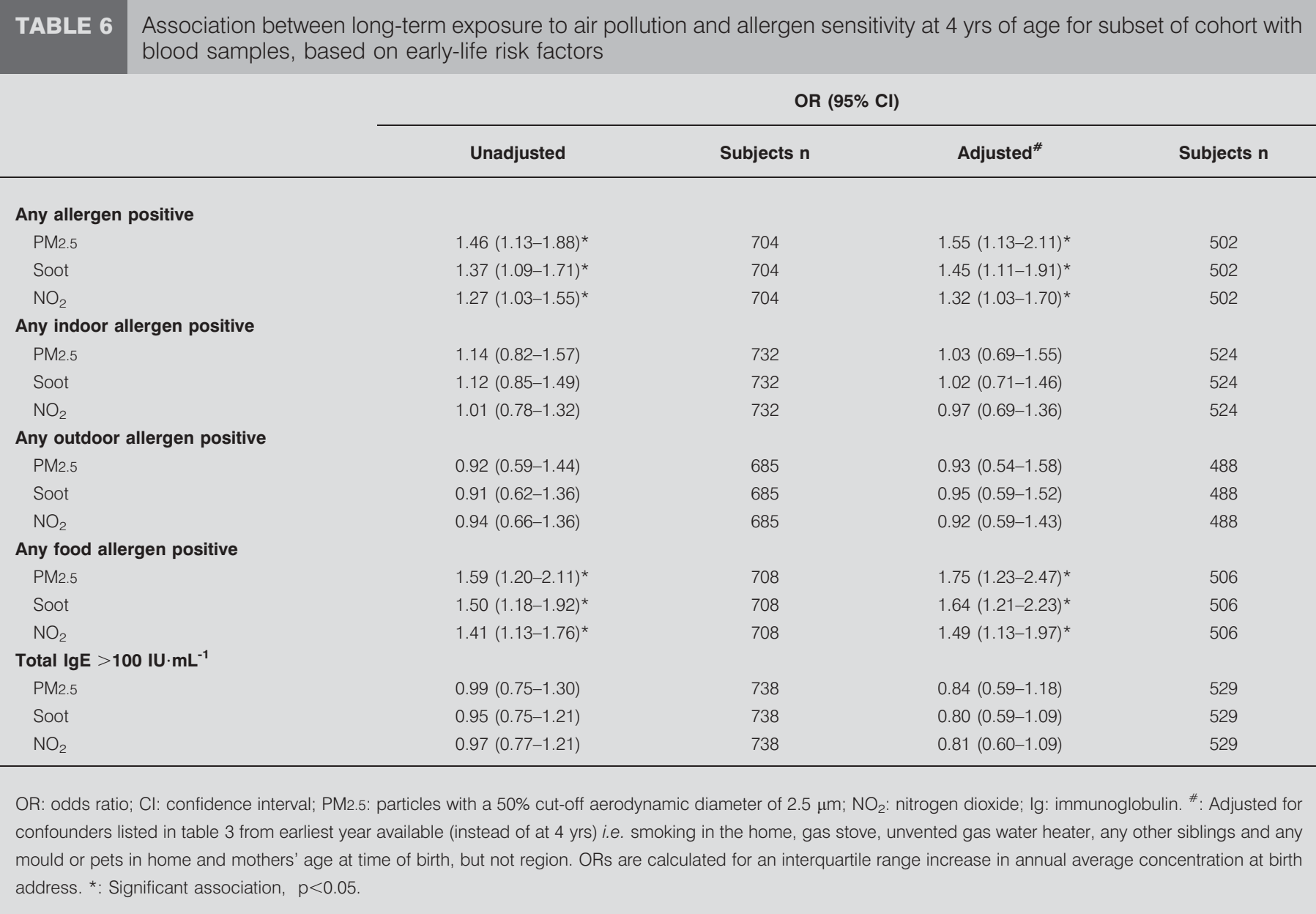

A limitation of the earlier analysis [26] relates to the validity of the specific health outcome variables for disease diagnosis at very young ages. Interestingly, the associations with wheeze reported earlier largely remained, whereas at 4 yrs of age the reporting of current wheeze can be assumed to have more validity as an indicator of asthma than at 2 yrs of age. This suggests that air pollutants contributed similarly to the incidence of the different phenotypes in early life. Future studies, including longitudinal analyses of the present cohort, will also help to reveal whether these early life exposures also affect asthma at older ages. Additionally, these analyses refer to health outcomes derived from questionnaires and responses that were not verified by objective measures in most cases. One exception is the demonstration in the present cohort, that the measure of physician-diagnosed asthma, but not reporting of wheeze, was being associated with higher levels of exhaled nitric oxide [36]. An additional limitation is that exposures are based upon outdoor concentrations estimated for the home address at birth and do not consider personal exposures directly. Recent work has, however, shown that personal exposures are related to measures of traffic proximity and broadly support the validity of the exposure assessment applied in the present study [37, 38]. Balanced against these limitations is the large size of the present birth cohort and the individual exposure level assessment.
The finding of a positive association between air pollution and objectively measured sensitisation to common allergens supports the findings of subjectively reported symptoms. Sensitisation data were available for a subgroup of the full cohort, with an overrepresentation of children born to allergic mothers. The association with sensitisation appeared to be limited to food allergens, whereas an association with indoor or outdoor aeroallergens seems more plausible. In early childhood, however, sensitisation manifests itself first as sensitisation towards food allergens, especially milk allergen. In the present cohort at $4 \mathrm{yrs}$ of age, the prevalence of food allergen sensitisation was about twice that of indoor allergens and more than three times that of outdoor allergens. Food allergy in early childhood may develop into allergy towards aeroallergens later. Therefore, it would be of interest to study whether the associations between air pollution and sensitisation shift towards aeroallergens at older ages. The low prevalence of (in particular) sensitisation to outdoor allergens limits the interpretation of the ORs, evidenced by the large confidence intervals. Surprisingly, the association of air pollution and total $\mathrm{IgE}$ did not support the association for sensitisation to any allergen. It has previously been reported that total $\operatorname{IgE}$ and $\operatorname{IgE}$ towards specific allergens are associated with different clinical presentations and may be determined by different mechanisms [39, 40]. 

TABLE 7 Association between exposure to air pollution and cumulative lifetime indicators of asthma and allergy status symptoms at 4 yrs of age for full cohort members with estimated exposures and completed yr-4 questionnaire, based on early-life risk factors

\section{OR $(95 \% \mathrm{Cl})$}

\begin{tabular}{|c|c|c|c|c|}
\hline & Unadjusted & Subjects $n$ & Adjusted $^{\#}$ & Subjects $n$ \\
\hline \multicolumn{5}{|c|}{ Wheeze-ever } \\
\hline PM2.5 & $1.15(1.02-1.31)^{*}$ & 3524 & $1.22(1.06-1.41)^{\star}$ & 2575 \\
\hline Soot & $1.13(1.02-1.26)^{*}$ & 3524 & $1.18(1.04-1.34)^{*}$ & 2575 \\
\hline $\mathrm{NO}_{2}$ & $1.11(1.00-1.23)^{*}$ & 3524 & $1.19(1.05-1.34)^{*}$ & 2575 \\
\hline \multicolumn{5}{|c|}{ Doctor-diagnosed asthma } \\
\hline PM2.5 & $1.17(0.95-1.45)$ & 3528 & $1.32(1.04-1.69)^{*}$ & 2575 \\
\hline Soot & $1.16(0.96-1.39)$ & 3528 & $1.26(1.02-1.56)^{*}$ & 2575 \\
\hline $\mathrm{NO}_{2}$ & $1.16(0.98-1.38)$ & 3528 & $1.28(1.04-1.56)^{*}$ & 2575 \\
\hline \multicolumn{5}{|c|}{ Probable asthma } \\
\hline PM2.5 & $1.18(1.01-1.38)^{*}$ & 3543 & $1.08(0.90-1.30)$ & 2588 \\
\hline Soot & $1.16(1.01-1.33)^{*}$ & 3543 & $1.06(0.90-1.24)$ & 2588 \\
\hline $\mathrm{NO}_{2}$ & $1.12(0.98-1.27)$ & 3543 & $1.04(0.89-1.22)$ & 2588 \\
\hline \multicolumn{5}{|c|}{ Early wheeze } \\
\hline PM2.5 & $1.05(0.92-1.19)$ & 3863 & $1.16(1.00-1.34)^{*}$ & 2588 \\
\hline Soot & $1.02(0.91-1.14)$ & 3863 & $1.11(0.97-1.26)$ & 2588 \\
\hline $\mathrm{NO}_{2}$ & $1.03(0.93-1.15)$ & 3863 & $1.13(1.00-1.28)^{*}$ & 2588 \\
\hline \multicolumn{5}{|c|}{ Persistent wheeze } \\
\hline PM2.5 & 1.19 (0.99-1.43) & 3863 & $1.19(0.96-1.48)$ & 2588 \\
\hline Soot & $1.17(1.00-1.38)^{*}$ & 3863 & $1.18(0.98-1.42)$ & 2588 \\
\hline $\mathrm{NO}_{2}$ & $1.10(0.94-1.28)$ & 3863 & $1.13(0.94-1.35)$ & 2588 \\
\hline \multicolumn{5}{|c|}{ Early frequent wheeze } \\
\hline $\mathrm{PM} 2.5$ & $1.15(0.96-1.39)$ & 3863 & $1.19(0.96-1.47)$ & 2588 \\
\hline Soot & $1.12(0.95-1.31)$ & 3863 & $1.14(0.95-1.37)$ & 2588 \\
\hline $\mathrm{NO}_{2}$ & $1.09(0.94-1.27)$ & 3863 & $1.14(0.95-1.36)$ & 2588 \\
\hline \multicolumn{5}{|c|}{ Doctor-diagnosed bronchitis } \\
\hline \multicolumn{5}{|c|}{ ever } \\
\hline PM2.5 & $0.99(0.86-1.14)$ & 3475 & $0.96(0.81-1.13)$ & 2543 \\
\hline Soot & $0.99(0.87-1.12)$ & 3475 & $0.95(0.82-1.10)$ & 2543 \\
\hline $\mathrm{NO}_{2}$ & $0.96(0.85-1.08)$ & 3475 & $0.94(0.82-1.08)$ & 2543 \\
\hline \multicolumn{5}{|c|}{ Itchy rash/eczema } \\
\hline PM2.5 & $1.04(0.93-1.16)$ & 3864 & $0.99(0.88-1.13)$ & 2588 \\
\hline Soot & $1.03(0.94-1.14)$ & 3864 & $0.99(0.89-1.11)$ & 2588 \\
\hline $\mathrm{NO}_{2}$ & $1.01(0.93-1.11)$ & 3864 & $0.98(0.88-1.09)$ & 2588 \\
\hline \multicolumn{5}{|c|}{$\begin{array}{l}\text { Doctor-diagnosed eczema } \\
\text { ever }\end{array}$} \\
\hline PM2.5 & $1.10(0.97-1.25)$ & 3464 & $0.98(0.85-1.13)$ & 2536 \\
\hline Soot & $1.09(0.98-1.22)$ & 3464 & $0.99(0.87-1.12)$ & 2536 \\
\hline $\mathrm{NO}_{2}$ & $1.06(0.95-1.17)$ & 3464 & $0.97(0.86-1.10)$ & 2536 \\
\hline
\end{tabular}

OR: odds ratio; $\mathrm{Cl}$ : confidence interval; PM2.5: particles with a $50 \%$ cut-off aerodynamic diameter of $2.5 \mu$ m; $\mathrm{NO}_{2}$ : nitrogen dioxide. ${ }^{\#}$ : Adjusted for confounders listed in table 3 from earliest year available (instead of at 4 yrs) i.e. smoking in the home, gas stove, unvented gas water heater, any other siblings and any mould or pets in home and mothers' age at time of birth but not region. ORs are calculated for an interquartile range increase in annual average concentration at birth address. *: Siginificant association, $p<0.05$

Early childhood respiratory effects may develop into chronic respiratory impairment later in life and, for this reason, it is important to identify modifiable determinants of adverse respiratory outcomes in the first years of life [41, 42].

The present analysis utilised a unique approach to calculate individual air pollution concentrations for the home addresses of each cohort member and, therefore, has distinct advantages over ecological analyses that assign single air pollution concentrations to all individuals living in certain areas [29]. In particular, the exposure model was developed to incorporate the impact of traffic sources on air pollution concentrations and therefore to capture the variability in exposure to traffic-related air pollution. This represents a major difference 
from the more typical use of government monitoring network data to estimate exposures in epidemiological studies. Most network monitors are located in order to measure urban or regional background air pollution concentrations, and thus to specifically avoid reflecting the influence of local traffic. As the cohort was drawn from throughout the Netherlands and included individuals living in urban and rural areas, there was a significant variability in exposure to traffic-related pollutants, but the magnitude of exposure differences was relatively small compared with between-city or longitudinal comparisons.

As in the present authors' earlier analysis [26], inconsistent differences were observed in associations with health outcomes between the three pollutant measures given the high correlation between the pollutants. The high correlations among the different pollutants that were measured precluded analysis of the impact of specific pollutants or indicators of specific components (e.g. heavy-duty vehicles) of traffic-related air pollution. The geographic variables, which were used to estimate exposure, were selected as surrogates for exposure to traffic sources in general.

In summary, the relationship between traffic-related air pollution and the development of asthmatic/allergic symptoms, respiratory infections and sensitisation was examined in a large birth cohort study $(n=\sim 4,000)$ in the Netherlands. A validated model was used to assign outdoor concentrations of traffic-related air pollutants (nitrogen dioxide, particles with a $50 \%$ cut-off aerodynamic diameter of $2.5 \mu \mathrm{m}$ and soot) at the home addresses of the cohort. Questionnaire-derived data on self-reported wheeze, dry night-time cough, ear/nose/throat infections, skin rash and physician diagnoses of asthma, bronchitis, influenza and eczema at 4 yrs of age were analysed in relation to air pollutants. Adjusted odds ratios for wheeze, doctor-diagnosed asthma, ear/nose/throat infections and flu/ serious colds indicated positive associations with air pollutants. No associations were observed for eczema and bronchitis. The findings at 4 yrs of age confirm earlier observations made at 2 yrs of age in the same cohort [26]. The finding of a positive association between air pollution and objectively measured sensitisation to common allergens supports the findings of subjectively reported symptoms. The association with sensitisation was limited to food allergens, with no indication of an association with indoor or outdoor aeroallergens, probably reflecting the low sensitisation prevalence at this age.

\section{REFERENCES}

1 Trasande L, Thurston GD. The role of air pollution in asthma and other pediatric morbidities. I Allergy Clin Immunol 2005; 115: 689-699.

2 von Mutius E. The environmental predictors of allergic disease. J Allergy Clin Immunol 2001; 105: 9-19.

3 Hwang BF, Lee YL, Lin YC, Jaakkola JJ, Guo YL. Traffic related air pollution as a determinant of asthma among Taiwanese school children. Thorax 2005; 60: 467-473.

4 McConnell R, Berhane K, Gilliland F, et al. Asthma in exercising children exposed to ozone: a cohort study. Lancet 2002; 359: 386-391.
5 Heinrich J, Wichmann HE. Traffic related pollutants in Europe and their effect on allergic disease. Curr Opin Allergy Clin Immunol 2004; 4: 341-348.

6 Brunekreef B, Sunyer J. Asthma, rhinitis and air pollution: is traffic to blame? Eur Respir J 2003; 21: 913-915.

7 Wjst M, Reitmeir P, Dold S, et al. Road traffic and adverse effects on respiratory health in children. BMJ 1993; 307: 596-600.

8 Oosterlee A, Drijver M, Lebret E, Brunekreef B. Chronic respiratory symptoms in children and adults living along streets with high traffic density. Occup Environ Med 1996; 53: 241-247.

9 Weiland SK, Mundt KA, Ruckmann A, Keil U. Selfreported wheezing and allergic rhinitis in children and traffic density on street of residence. Ann Epidemiol 1994; 4: 243-247.

10 English P, Neutra R, Scalf R, Sullivan M, Waller L, Zhu L. Examining associations between childhood asthma and traffic flow using a geographic information system. Environ Health Perspect 1999; 107: 761-767.

11 Hirsch T, Weiland SK, von Mutius E, et al. Inner city air pollution and respiratory health and atopy in children. Eur Respir J 1999; 14: 669-677.

12 Kim JJ, Smorodinsky S, Lipsett M, Singer BC, Hodgson AT, Ostro B. Traffic-related air pollution near busy roads: the East Bay Children's Respiratory Health Study. Am J Respir Crit Care Med 2004; 170: 520-526.

13 Shima M, Nitta Y, Adachi M. Traffic-related air pollution and respiratory symptoms in children living along trunk roads in Chiba Prefecture, Japan. J Epidemiol 2003; 13: 108-119.

14 Venn AJ, Lewis SA, Cooper M, Hubbard R, Britton J. Living near a main road and the risk of wheezing illness in children. Am J Respir Crit Care Med 2001; 164: 2177-2180.

15 Zmirou D, Gauvin S, Pin I, et al. Traffic related air pollution and incidence of childhood asthma: results of the Vesta case-control study. J Epidemiol Community Health 2004; 58: $18-23$.

16 Pershagen G, Rylander E, Norberg S, Eriksson M, Nordvall SL. Air pollution involving nitrogen dioxide exposure and wheezing bronchitis in children. Int $J$ Epidemiol 1995; 24: 1147-1153.

17 Edwards J, Walters S, Griffiths RK. Hospital admissions for asthma in pre-school children: relationship to major roads in Birmingham, United Kingdom. Arch Environ Health 1994; 49: 223-227.

18 McConnell R, Berhane K, Yao L, et al. Traffic, susceptibility, and childhood asthma. Environ Health Perspect 2006; 114: 766-772.

19 Gauderman WJ, Avol E, Lurmann F, et al. Childhood asthma and exposure to traffic and nitrogen dioxide. Epidemiology 2005; 16: 737-743.

20 Nicolai T, Carr D, Weiland SK, et al. Urban traffic and pollutant exposure related to respiratory outcomes and atopy in a large sample of children. Eur Respir J 2003; 21: 956-963.

21 Brunekreef B, Janssen NA, de Hartog J, Harssema H, Knape M, van Vliet P. Air pollution from truck traffic and lung function in children living near motorways. Epidemiology 1997; 8: 298-303.

22 van Vliet P, Knape M, de Hartog J, Janssen N, Harssema H, Brunekreef B. Motor vehicle exhaust and chronic respira- 
tory symptoms in children living near freeways. Environ Res 1997; 74: 122-132.

23 Janssen NA, Brunekreef B, van Vliet $\mathrm{P}$, et al. The relationship between air pollution from heavy traffic and allergic sensitization, bronchial hyperresponsiveness, and respiratory symptoms in Dutch schoolchildren. Environ Health Perspect 2003; 111: 1512-1518.

24 Braun-Fahrlander C, Ackermann-Liebrich U, Schwartz J, Gnehm HP, Rutishauser M, Wanner HU. Air pollution and respiratory symptoms in preschool children. Am Rev Respir Dis 1992; 145: 42-47.

25 von Mutius E, Sherrill DL, Fritzsch C, Martínez FD, Lebowitz MD. Air pollution and upper respiratory symptoms in children from East Germany. Eur Respir J 1995; 8: 723-728.

26 Brauer M, Hoek G, Van Vliet P, et al. Air pollution from traffic and the development of respiratory infections and asthmatic and allergic symptoms in children. Am J Respir Crit Care Med 2002; 166: 1092-1098.

27 Wijga A, Smit HA, Brunekreef B, et al. Are children at high familial risk of developing allergy born into a low risk environment? The PIAMA Birth Cohort Study. Prevention and Incidence of Asthma and Mite Allergy. Clin Exp Allergy 2001; 31: 576-581.

28 Koopman LP, Smit HA, Heijnen ML, et al. Respiratory infections in infants: interaction of parental allergy, childcare, and siblings - The PIAMA study. Pediatrics 2001; 108: 943-948.

29 Brauer M, Hoek G, van Vliet P, et al. Estimating long-term average particulate air pollution concentrations: application of traffic indicators and geographic information systems. Epidemiology 2003; 14: 228-239.

30 Lewné M, Cyrys J, Meliefste K, et al. Spatial variation in nitrogen dioxide in three European areas. Sci Total Environ 2004; 332: 217-230.

31 Hoek G, Meliefste K, Cyrys J, et al. Spatial variability of fine particle concentrations in three European countries. Atmos Environ 2002; 36: 4077-4088.
32 Gehring U, Cyrys J, Sedlmeir G, et al. Traffic-related air pollution and respiratory health during the first 2 yrs of life. Eur Respir J 2002; 19: 690-698.

33 Cyrys J, Heinrich J, Hoek G, et al. Comparison between different traffic-related particle indicators: elemental carbon (EC), PM2.5 mass, and absorbance. J Expo Anal Environ Epidemiol 2003; 13: 134-143.

34 Weiland SK, Bjorksten B, Brunekreef B, Cookson WO, von Mutius E, Strachan DP. Phase II of the International Study of Asthma and Allergies in Childhood (ISAAC II): rationale and methods. Eur Respir J 2004; 24: 406-412.

35 Brussee JE, Smit HA, van Strien RT, et al. Allergen exposure in infancy and the development of sensitization, wheeze, and asthma at 4 years. J Allergy Clin Immunol 2005; 115: 946-952.

36 Brussee JE, Smit HA, Kerkhof M, et al. Exhaled nitric oxide in 4-year-old children: relationship with asthma and atopy. Eur Respir J 2005; 25: 455-461.

37 Wichmann J, Janssen NAH, van der Zee S, Brunekreef B. Traffic-related differences in indoor and personal absorption coefficient measurements in Amsterdam, the Netherlands. Atmos Environ 2005; 39: 7384-7392.

38 van Roosbroeck S, Wichmann J, Janssen NA, et al. Longterm personal exposure to traffic-related air pollution among school children, a validation study. Sci Total Environ 2006; 368: 565-573.

39 Sunyer J, Anto JM, Castellsague J, Soriano JB, Roca J. Total serum IgE is associated with asthma independently of specific IgE levels. The Spanish Group of the European Study of Asthma. Eur Respir J 1996; 9: 1880-1884.

40 Sinclair D, Peters SA. The predictive value of total serum IgE for a positive allergen specific IgE result. J Clin Pathol 2004; 57: 956-959.

41 Lau S, Nickel R, Niggemann B, et al. The development of childhood asthma: lessons from the German Multicentre Allergy Study (MAS). Paediatr Respir Rev 2002; 3: 265-272.

42 Martínez FD. What have we learned from the Tucson Children's Respiratory Study? Paediatr Respir Rev 2002; 3: 193-197. 